\title{
Local spin-flip spectral distribution obtained by resonant x-ray Raman scattering
}

\author{
F. M. F. de Groot \\ Solid State Physics, University of Groningen, Nijenborgh 4, 9747 AG Groningen, The Netherlands \\ P. Kuiper \\ Department of Physics, Uppsala University, Box 530, S-75121, Uppsala, Sweden \\ G. A. Sawatzky \\ Solid State Physics, University of Groningen, Nijenborgh 4, 9747 AG Groningen, The Netherlands
}

(Received 17 February 1998)

\begin{abstract}
We show that resonant x-ray Raman (RXR) scattering can be used to study the local spin-flip excitation spectral distribution in magnetically ordered $3 d$ transition-metal compounds. We demonstrate with realistic calculations on the $3 p$ x-ray absorption edge of $\mathrm{a} \mathrm{Cu}^{2+}$ compound and the $2 p$ edge of a $\mathrm{Ni}^{2+}$ compound that the scattered $x$-ray energy and intensity distribution contains the excitation spectrum resulting from a single local spin flip, as well as $d d$ excitations accompanied by local spin flip satellites. We develop the theory describing this effect and discuss the polarization conditions that can be used to observe these effects. [S0163-1829(98)02320-0]
\end{abstract}

Recent advances in synchrotron radiation sources highresolution monochromators and x-ray detection techniques have made high-resolution $\mathrm{x}$-ray scattering techniques useful in the study of phonons and crystal field excitations in solids. ${ }^{1,2}$ In this paper we show that there is a way of obtaining information on the local spin-flip excitations using specific resonances involving core orbitals with a large spin orbit coupling. Such resonances are extremely strong at the $3 d$ transition metal $2 p$ and $3 p$ edges as well as the $3 d$ and $4 d$ edges of the rare earths. Since these elements form the basis of most magnetic materials the use of such techniques could prove to be very important. The basic idea is to use the large spin orbit coupling of the core hole in the intermediate states in a resonant $\mathrm{X}$-ray Raman (RXR) scattering experiment to study excitations in which only the local valence electron spin has been flipped. This relates closely to spin-flip Raman scattering in the optical energy range, a technique used since the 1970 s. ${ }^{3,4}$ In light scattering a series of spin-dependent effects are known to affect the (resonant) Raman scattering. Spin-dependent scattering due to the simultaneous excitation of the spin and phonon systems has been studied often. ${ }^{4}$ In systems with an accessible spin-orbit splitting in the valence band, this spin-orbit splitting has been used directly to create pure spin-flip transitions. ${ }^{3}$ In the present paper the basic mechanism is the same: a spin-orbit split state is used to create a local spin-flip transition. The use of core levels, however, makes the technique general for all elements and not sensitive to the details of the spin-orbit splitting of the valence band, being applicable to systems where this is negligible as the $3 d$ transition-metal compounds. In the case of optical Raman spectra they commonly probe the two magnon excitations, for which the line shape is still a matter of controversy. ${ }^{5}$ This, combined with the well-known advantages of resonant $\mathrm{X}$-ray techniques, such as element specificity, will make RXR scattering an important technique to study exchange interactions in magnetic materials. In a magnetically ordered solid the spin flip of a local valence elec- tron spin provides direct information on the exchange or superexchange interactions with neighboring atoms in the ground state. The spectral shape at $T=0$ will be that of a local magnon density of states and at finite temperatures the energy will measure the average local exchange field, which in a mean-field approximation would be directly related to the average temperature-dependent magnetic moment.

In this paper we investigate the feasibility of such experiments and show that under now attainable experimental conditions the experiments are possible. We discuss the physics involved and the relation between the polarization conditions and the information to be expected. To demonstrate the effect we use divalent $\mathrm{Cu}$ in octahedral and tetragonal crystal fields as well as divalent $\mathrm{Ni}$ in octahedral coordination. To simulate the effect of exchange interactions with neighboring magnetic ions we add a magnetic field with a magnitude resulting in comparable Zeeman splittings. This would be equivalent to replacing the Heisenberg spin Hamiltonian by a mean-field approximation, which is sufficient for our purposes to describe the effect and the information one can obtain. The magnetic field one requires is of course very large since the (super)exchange interactions are as high as $0.12 \mathrm{eV}$ (Ref. 5) in the high- $T_{c}$ cuprates resulting in a total spin flip energy of a local spin of $0.25 \mathrm{eV}$.

We start with the simplest of cases, namely, that of RXR scattering at the $\mathrm{Cu} 3 p$ edge in divalent $\mathrm{Cu}$ compounds. The case of $\mathrm{Cu}^{2+}\left(3 d^{9}\right)$ is very simple as only a single hole is present in the initial, intermediate, and final states. The intermediate is spin orbit split into a $j=3 / 2$ and a $j=1 / 2$ state, which makes a spin-flip transition possible. In the final state the core hole is again filled by a valence $d$ electron resulting in a $3 d^{9}$ configuration but possibly in an excited state. This excited state could be merely a spin flip in the field of the surroundings, which can result only if there is a compensating change in the angular momentum (polarization) of the photon. An effect that increases the sensitivity of such a measurement is that for orthogonal polarizations of incident 
TABLE I. Single particle matrix elements for a $p d$ transition. All matrix elements have been multiplied with $\sqrt{30}$. Left circular polarization is indicated with italic, right circular polarization with roman and $z$ polarization with boldface. The $3 d$ spin-orbit coupling is neglected. The six intermediate states have been separated into their constituting spin-up and spin-down parts at the bottom, where $\alpha=\sqrt{2 / 3}$ and $\beta=\sqrt{1 / 3}$.

\begin{tabular}{lcccccc}
\hline \hline & $\left|\frac{3}{2}, \frac{3}{2}\right\rangle$ & $\left|\frac{3}{2}, \frac{1}{2}\right\rangle$ & $\left|\frac{3}{2},-\frac{1}{2}\right\rangle$ & $\left|\frac{3}{2},-\frac{3}{2}\right\rangle$ & $\left|\frac{1}{2}, \frac{1}{2}\right\rangle$ & $\left|\frac{1}{2},-\frac{1}{2}\right\rangle$ \\
\hline$Y_{22}(\downarrow)$ & & $+\sqrt{2}$ & & & $+\sqrt{4}$ & \\
$Y_{21}(\downarrow)$ & & $+\sqrt{\mathbf{1}}$ & $+\sqrt{2}$ & & $+\sqrt{\mathbf{2}}$ & $-\sqrt{1}$ \\
$Y_{20}(\downarrow)$ & & $-\sqrt{1 / 3}$ & $-\sqrt{\mathbf{8 / 3}}$ & $+\sqrt{1}$ & $-\sqrt{2 / 3}$ & $+\sqrt{\mathbf{4 / 3}}$ \\
$Y_{2 \overline{1}}(\downarrow)$ & & & $+\sqrt{2}$ & $-\sqrt{\mathbf{3}}$ & & $-\sqrt{1}$ \\
$Y_{22}(\downarrow)$ & & & & $+\sqrt{6}$ & & \\
$Y_{22}(\uparrow)$ & $-\sqrt{6}$ & & & & & \\
$Y_{21}(\uparrow)$ & $+\sqrt{\mathbf{3}}$ & $+\sqrt{2}$ & & & $-\sqrt{1}$ & \\
$Y_{20}(\uparrow)$ & $+\sqrt{1}$ & $+\sqrt{\mathbf{8} / \mathbf{3}}$ & $+\sqrt{1 / 3}$ & & $-\sqrt{\mathbf{4 / 3}}$ & $+\sqrt{2 / 3}$ \\
$Y_{21}(\uparrow)$ & & $-\sqrt{2}$ & $-\sqrt{\mathbf{1}}$ & & $+\sqrt{1}$ & $-\sqrt{\mathbf{2}}$ \\
$Y_{22}(\uparrow)$ & & & $+\sqrt{2}$ & & & $+\sqrt{4}$ \\
& $Y_{11}(\uparrow)$ & $\alpha Y_{10}(\uparrow)$ & $\beta Y_{1 \overline{1}}(\uparrow)$ & & $\beta Y_{10}(\uparrow)$ & $\alpha Y_{1 \overline{1}}(\uparrow)$ \\
& & $\beta Y_{11}(\downarrow)$ & $\alpha Y_{10}(\downarrow)$ & $Y_{1 \overline{1}}(\downarrow)$ & $\alpha Y_{11}(\downarrow)$ & $\beta Y_{10}(\downarrow)$ \\
\hline \hline
\end{tabular}

and scattered photon elastic scattering intensity vanishes. The RXR scattering intensity is given by the KramersHeisenberg formula: ${ }^{6}$

$$
I_{\left(\hbar \omega, \hbar \omega^{\prime}\right)} \propto \sum_{q^{\prime}, q} F_{q^{\prime} q} \delta_{E_{f}-E_{0}+\hbar \omega^{\prime}-\hbar \omega}
$$

with

$$
F_{q^{\prime} q}=\sum_{3 d}\left|\sum_{3 p} \frac{\left\langle 3 d\left|C_{q^{\prime}}^{(1)}\right| 3 p\right\rangle\left\langle 3 p\left|C_{q}^{(1)}\right| 3 d_{0}\right\rangle}{E_{3 d_{0}}+\hbar \omega-E_{3 p}-i \Gamma_{3 p}}\right|^{2}
$$

The dipole matrix elements $\left(C_{q}\right)$ to the $3 p^{5}$ states are calculated for all polarizations $(q)$. The $M_{2,3}$ edge consist of two peaks, separated by the $3 p$ spin-orbit splitting of about $2.5 \mathrm{eV}$. The $3 p$ lifetime broadening is approximately $0.4 \mathrm{eV}$ full width at half maximum (FWHM). Note that the core hole lifetime broadening does not influence the resolution or linewidth because of the coherence of the process. The lifetime of the spin flipped final states will be given by the spin lattice relaxation time and the spectral shape and width will display the magnon density of states.

If the $3 d$ spin-orbit coupling is neglected the $3 p \rightarrow 3 d$ matrix elements are given by the single-electron $p \rightarrow d$ matrix elements. ${ }^{7}$ They are given in Table I, which concentrates on the division into spin up and spin down of the intermediate states. In order to see which final states can be reached starting with a single hole in a $d$ orbital, one should look at Eq. (1) and first determine the possible intermediate states one can go to by selecting the ground-state $d$ orbital in the left column and moving to the right. The transition amplitudes and polarizations are then read from the table. To then see which final states can be reached pick a particular intermediate state reached with the used polarization from the top row and move down reading of the final $d$ states reachable together with the polarization and amplitude of the outgoing photon.
We see that different combinations of polarizations of in and out photons result in the possibility of reaching almost any final state one likes, which will be shown more clearly below. Concentrating on the idea that spin flip excitations can be especially observed, we note that there are many possible paths in which we go from an initial state in the upper half (spin down) to the lower half (spin up) in the final state. These represent possible spin flip excitations that may or may not be accompanied by a change in the orbital part of the $d$ wave function. We also see there are paths for which the orbital part does not change and only a spin flip occurs. For example, spin-down $Y_{20}$, i.e., the $d_{3 z^{2}-r^{2}}$ orbital, can scatter to the $|1 / 2,1 / 2\rangle$ state with $q=1$ and then to spin-up $Y_{20}$ with $q=0$. The other states reached in this process will have higher energies because they involve crystal field excitations in the actual solid. The reason this spin flip happens is of course due to the fact that the $j=3 / 2$ and $j=1 / 2$ states in the intermediate state are separated by the spin-orbit coupling and the spin is no longer a good quantum number. We also see in the example given that the total angular momentum is conserved because of the change in the angular moment of the spin by +1 is compensated by a change in the angular momentum of the photons by -1 . It turns out that it is more suitable and also more feasible at least on the detection side to use linear polarization rather than circular. The table given above can easily be converted to this case. Since in the systems we will study the symmetry is either cubic or tetragonal with the spin along the $z$ axis and perpendicular to the tetragonal plane, $x$ and $y$ are equivalent so that we need only study polarization combinations of $x$ in $x$ out and $x$ in $z$ out. The spectra at other arbitrary rotations can easily be determined.

We note that for the analysis it makes no difference if the system is in a ferromagnetic or an antiferromagnetic state, which is another advantage of using linear polarized light. For example, an antiferromagnet has both $d_{x^{2}-y^{2}}^{\uparrow}$ and $d_{x^{2}-y^{2}}^{\downarrow}$ states, but locally the spin in the excited final states are also reversed. A difference is that the scattering of left and right circularly polarized light is reversed with the result that the MCD effect will disappear. What does not disappear is the difference in the $F_{x x}$ and $F_{z x}$ scattering. Important here is that even if the detection cannot be done with polarization selection, an appropriate choice of the scattering angles with a given incident polarization can allow for some polarization selection to be made.

The concepts outlined above will be used to calculate the RXR spectra of $\mathrm{Cu}^{2+}$ in octahedral and tetragonal symmetry, and of $\mathrm{Ni}^{2+}$ in octahedral symmetry. Figure 1(a) shows the RXR spectra for a $\mathrm{Cu}^{2+}$ ion in octahedral symmetry in an antiferromagnet. In the calculation an octahedral crystal field of $1.0 \mathrm{eV}$ and an exchange field of $0.25 \mathrm{eV}$, acting on the spin only, have been included. This gives the $e_{g}(\downarrow)$ states at zero energy, the $e_{g}(\uparrow)$ states at $0.25 \mathrm{eV}$, the $t_{2 g}(\downarrow)$ states at about $1.0 \mathrm{eV}$ (split by the $3 d$ spin-orbit coupling), and the $t_{2 g}(\uparrow)$ states at $1.25 \mathrm{eV}$. The experimental broadenings have been approximated with a Gaussian of $0.1 \mathrm{eV}$ FWHM. The total scattering has been normalized to the same height for all 3 energies.

This simple example contains two important effects: (1) $F_{x x}$ scattering has an elastic peak and transitions to the $t_{2 g}$ 

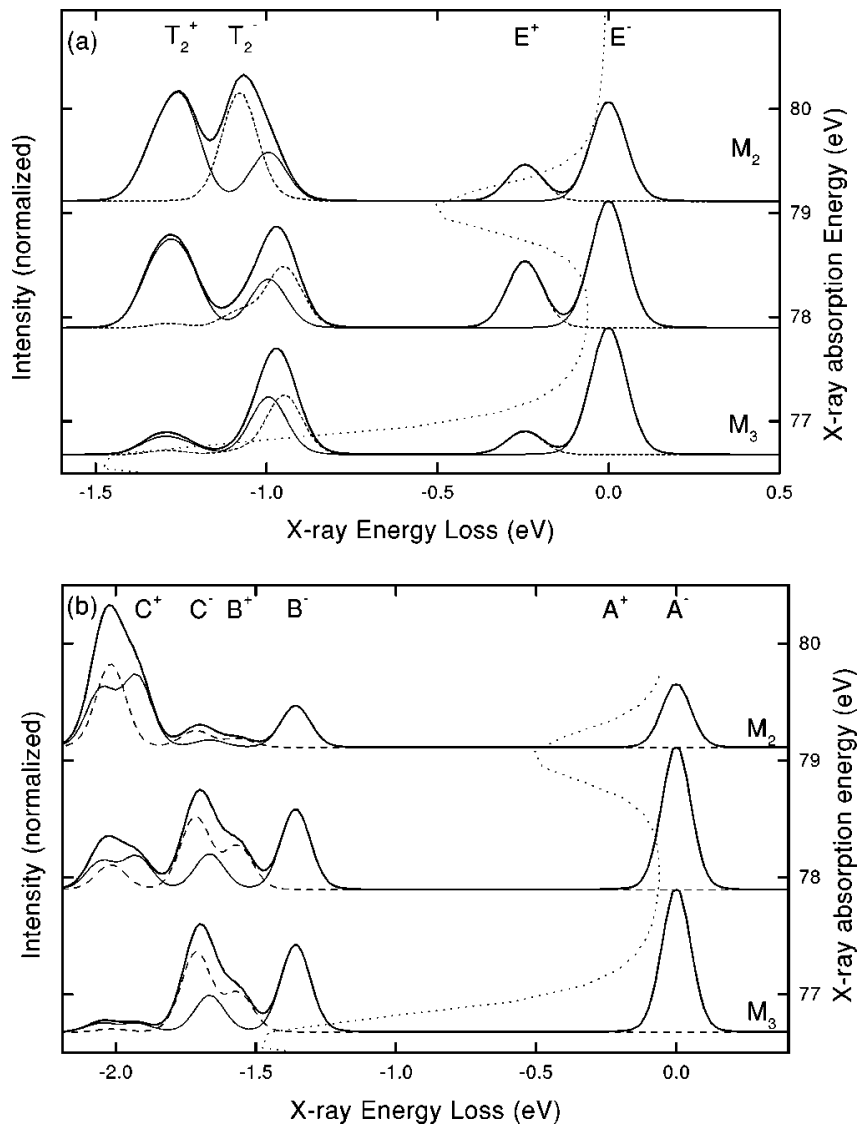

FIG. 1. RXR scattering at the $3 p$ edge of a $\mathrm{Cu}^{2+}$ system. (a) in octahedral symmetry: $\mathbf{E}$ denotes the $e_{g}$ states, $\mathbf{T}_{2}$ the $t_{2 g}$ states. (b) in tetragonal symmetry, using the crystal field parameters of $\mathrm{Sr}_{2} \mathrm{CuO}_{2} \mathrm{Cl}_{2}$ : A denotes $x^{2}-y^{2}$ states, $\mathbf{B} x y$ states, and $\mathbf{C} x z, y z$, and $z^{2}$ states. Note the absence of $A^{+}$scattering (see text). Indicated are the $F_{x x}$ scattering (solid), $F_{z x}$ scattering (dashed), and the total scattering (thick solid). The $\mathrm{x}$-ray-absorption spectrum is given with dots. The normalized RXR spectra are given at the respective x-ray absorption energies as indicated on the right.

states, including spin-flip transition at $1.25 \mathrm{eV}$, while $F_{z x}$ scattering does not show an elastic peak but contains the spin-flip transition at $0.25 \mathrm{eV}$, plus transitions to $t_{2 g}$ states. Thus with $F_{z x}$ scattering one can observe directly the spinflip transition of the ground state. (2) The spin-flip peaks have a relatively larger intensity at the $M_{2}$ edge. The reason is that the $M_{3}$ edge contains scattering via $|3 / 2,3 / 2\rangle$ and $|3 / 2,-3 / 2\rangle$ states, which do not allow spin-flip transitions if the moments are in the direction of the x-ray polarization (cf. Table I.). By making use of different excitation energies and of the angular dependences, detailed information can be obtained.

Figure 1(b) shows the same spectra for the $\mathrm{Cu}^{2+}$ ions in $\mathrm{Sr}_{2} \mathrm{CuO}_{2} \mathrm{Cl}_{2}$. The local symmetry is tetragonal; the $z^{2}$ states, as well as the $x z$ and $y z$ states, can be found at approximately $1.7 \mathrm{eV}$ and the $x y$ states at $1.35 \mathrm{eV}{ }^{8}$ The $3 d$ spinorbit coupling of $0.1 \mathrm{eV}$ and an exchange field of $0.25 \mathrm{eV}$ have been included. Peaks are observed at the excitation energies related to the different $3 d$ orbitals as indicated in the figure. However, it is found that the direct spin-flip peak related to the $x^{2}-y^{2}(\uparrow)$ state at $0.25 \mathrm{eV}$ is absent. The reason is that the $x^{2}-y^{2}$ state, a linear combination of atomic $Y_{22}$ and $Y_{2 \overline{2}}$ states, does not allow a direct spin-flip transition (cf.

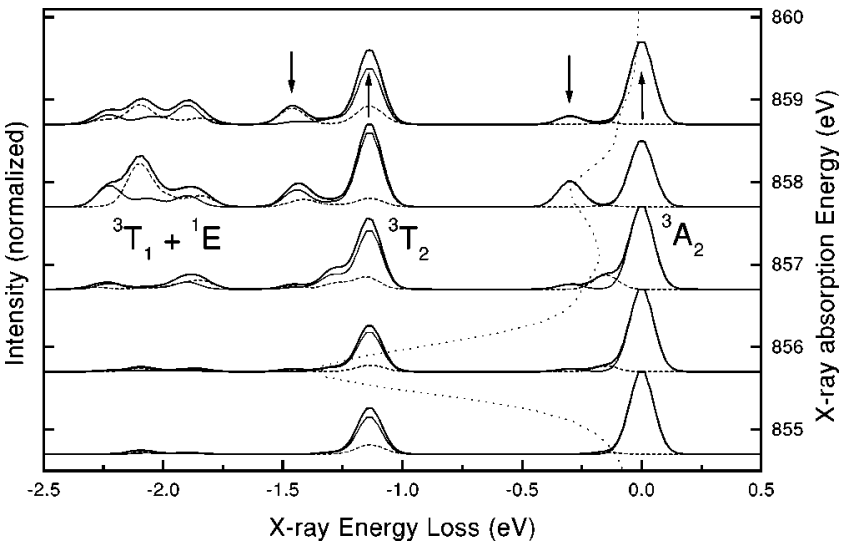

FIG. 2. RXR scattering at the $2 p$ edge of a $\mathrm{Ni}^{2+}$ system in octahedral symmetry, using the crystal-field parameter of $\mathrm{NiO}$. Indicated are $F_{x x}$ scattering (thin solid), $F_{z x}$ scattering (dashed), and the total scattering (thick solid). The x-ray-absorption spectrum is given with dots. The normalized RXR spectra are given at the respective $\mathrm{X}$-ray-absorption energies (in steps of $1.0 \mathrm{eV}$ ) as indicated on the right. The symmetries of the states are given at the middle spectrum. The $m_{s}$ substates are indicated at the top for spin up ( $\uparrow$ ) and spin down $(\downarrow)$.

Table I). It has been confirmed in experiment that $\mathrm{Sr}_{2} \mathrm{CuO}_{2} \mathrm{Cl}_{2}$ has a pronounced (and different) angular dependence of the peaks at 1.3 and $1.7 \mathrm{eV}$. The effects of the exchange field are confirmed by the additional intensity at higher energy at the $M_{2}$ edge. ${ }^{8}$ Transition-metal systems with less than nine $3 d$ electrons are constrained by strong multiplet effects. ${ }^{9}$ For example, $\mathrm{Ni}^{2+}$ has a $3 d^{8}$ ground state and a $2 p^{5} 3 d^{9}$ intermediate state. ${ }^{10}$

Figure 2 shows the RXR spectra of $\mathrm{Ni}^{2+}$ in octahedral symmetry with a crystal field of $1.13 \mathrm{eV}$ as observed in $\mathrm{NiO}{ }^{11}$ An exchange field of $0.15 \mathrm{eV}$ (in the propagation direction of the $\mathrm{x}$ ray) has been added. Indicated are the lowest $2.5 \mathrm{eV}$ of the RXR spectra, excited around the $L_{3}$ $\mathrm{x}$-ray absorption edge. The ground state has ${ }^{3} A_{2}$ symmetry, the first excited state has ${ }^{3} T_{2}$ symmetry and is found at 1.13 $\mathrm{eV}$. Around $2.0 \mathrm{eV}$ the ${ }^{3} T_{1}$ and ${ }^{1} E$ states are found and, not shown, still at higher energies all other $3 d^{8}$ multiplet states. We concentrate on ${ }^{3} A_{2}$ and ${ }^{3} T_{2}$, which are split by the exchange field into their $m_{s}=+1, m_{s}=0$, and $m_{s}=-1$ Zeeman states. At the $L_{3}$ edge $(855.7 \mathrm{eV})$ one observes the elastic peak and crystal field excitations to ${ }^{3} T_{2}\left(m_{s}=-1\right)$. Note that the elastic peak has pure $F_{x x}$ scattering, while the ${ }^{3} T_{2}$ peak is dominated by $F_{z x}$ scattering and will have a different angular dependence. Interesting things happen if the excitation energy is chosen at energies above the $L_{3}$ edge. At 856.7 $\mathrm{eV}$, the direct spin-flip peak to the $m_{s}=0$ state at $0.15 \mathrm{eV}$ is visible in $F_{z x}$ scattering, while the $m_{s}=0$ state is also visible for the ${ }^{3} T_{2}$ peak. In the case of the ${ }^{3} A_{2}$ peak, $m_{l}=0$, hence $m_{s}=m_{j}$. This explains why the $m_{s}=0$ peak is only seen in $F_{z x}$ scattering: $F_{z x}$ scattering relates to $\Delta m_{j}= \pm 1$ transitions, while $F_{x x}$ scattering relates to $\Delta m_{j}=0, \pm 2$ transitions. This selection rule can also be observed at $857.7 \mathrm{eV}$ excitation energy, where the transition to $m_{s}=+1$ becomes very pronounced and the ${ }^{3} A_{2}\left(m_{s}=+1\right)$ has pure $F_{x x}$ scattering. In the case of the ${ }^{3} T_{2}$ peak there is also an orbital momentum in play and the identity of $m_{s}$ and $m_{j}$ disappears. The $m_{s}=+1$ states have an energy of two times the exchange 
field and they should be clearly observable in experiment. Within the present paper, we wish to make an important conclusion that the spin-flip transitions can be studied in great detail, if the correct excitation energies are used in the RXR scattering experiments. We note further that ferromagnetic systems will additionally show significant MCD effects.

In this paper we have shown that RXR scattering involving a core hole intermediate state with strong spin orbit coupling is capable of exciting single local spin flip excitations. This provides a way of determining the interatomic exchange and superexchange interactions and more generally the local single magnon density of states. We have discussed the polarization conditions required and have shown that under certain conditions the elastic peak can be eliminated, providing a way of isolating the spin flip excitation even if the resolution is not really sufficient to resolve it from the elastically scattered beam. We have demonstrated the effect with detailed calculations of the RXR scattering line shape in divalent $\mathrm{Cu}$ and $\mathrm{Ni}$ compounds. Although such measurements are possible at present the improved resolution on the detection side in the near future together with the intense synchrotron light sources will permit a detailed study of the site selected local magnon density of states in ferromagnets and antiferromagnets as a function of temperature.

We thank Laurent Duda and Michel van Veenendaal for discussions and useful suggestions. The research of F.d.G. has been made possible by the Royal Netherlands Academy of Arts and Sciences (KNAW).
${ }^{1}$ F. Sette, G. Ruocco, M. Krisch, C. Masciovecchio, R. Verbeni, and U. Bergmann, Phys. Rev. Lett. 77, 83 (1996).

${ }^{2}$ S.M. Butorin, J.H. Guo, M. Magnuson, P. Kuiper, and J. Nordgren, Phys. Rev. B 54, 4405 (1996).

${ }^{3}$ G. Abstreiter et al., in Light Scattering in Solids, edited by M. Cardona and G. Güntherodt (Springer, Berlin, 1984), Vol. IV, Chap. 2.

${ }^{4}$ G. Güntherodt and R. Zeyher, in Light Scattering in Solids (Ref. 3), Chapter 4.

${ }^{5}$ J.D. Perkins et al., Phys. Rev. Lett. 71, 1621 (1993); J. Lorenzana and G.A. Sawatzky, ibid. 74, 1867 (1995).
${ }^{6}$ T. Åberg and B. Crasemann, in X-ray Anomalous (Resonance) Scattering: Theory and Experiment, edited by K. Fisher, G. Materlik, and C. Sparks (Elsevier, Amsterdam, 1994).

${ }^{7}$ N.V. Smith, C.T. Chen, F. Sette, and L.F. Mattheiss, Phys. Rev. B 46, 1023 (1992).

${ }^{8}$ P. Kuiper, J. Guo, C. Sathe, L. Duda, J. Nordgren, H. Pothuizen, F.M.F. de Groot, and G.A. Sawatzky (unpublished).

${ }^{9}$ S. Sugano, Y. Tanabe, and H. Kitamura, Multiplets of Transition Metal Ions (Academic Press, New York, 1970).

${ }^{10}$ F.M.F. de Groot, J. Electron Spectrosc. Relat. Phenom. 67, 529 (1994).

${ }^{11}$ R. Newman and R.M. Chrenko, Phys. Rev. 114, 1507 (1959). 